(c) American Dairy Science Association, 2007.

\title{
Short Communication: Patterns of Fecal Shedding of Klebsiella by Dairy Cows
}

\author{
M. A. Munoz and R. N. Zadoks ${ }^{1}$ \\ Quality Milk Production Services, Cornell University, Ithaca, NY 14850-1263
}

\begin{abstract}
Patterns and persistency of fecal shedding of Klebsiella spp. by healthy adult dairy cattle were explored with probabilistic, statistical, and molecular methods. Fecal shedding was monitored longitudinally in 92 animals in 1 herd for 5 mo. Shedding patterns followed a random binomial distribution, and associations with host factors were not detected. For 12 animals from 4 herds, strain-typing of multiple fecal Klebsiella isolates was performed by means of random-amplified polymorphic DNA typing. For 2 animals, additional typing was performed on isolates from samples collected on several consecutive days. A large variety of Klebsiella strains was detected within samples (on average, 3.1 strains per 4 isolates) and between samples (18 of 20 strains were detected only once in feces from cows that were sampled for $5 \mathrm{~d}$ consecutively). Results from each method suggest that fecal shedding of Klebsiella is associated with transient rather than persistent presence of the organism in the gastrointestinal tract.
\end{abstract}

Key words: Klebsiella, dairy cow, fecal shedding

Gram-negative bacteria are the most common etiological agents isolated from cases of clinical mastitis. The most common gram-negative bacteria causing mastitis are Escherichia coli and Klebsiella spp. (Hogan and Smith, 2003). Milk losses and mortality due to Klebsiella mastitis are higher compared with those resulting from $E$. coli mastitis (Gröhn et al., 2004). Vaccines and antibiotic therapy have limited effect against Klebsiella mastitis (Hogan and Smith, 2003; Roberson et al., 2004). Therefore, Klebsiella mastitis control is primarily based on prevention. Use of inorganic bedding material and good bedding management are the main preventive measures (Kristula et al., 2005). A recent study showed that fecal shedding of Klebsiella could occur in as many as $80 \%$ of healthy dairy cows (Munoz et al., 2006). This suggests that fecal shedding of the organism may contribute to the exposure of cows to Klebsiella

Received September 19, 2006.

Accepted November 1, 2006.

${ }^{1}$ Corresponding author: rz26@cornell.edu and to occurrence of mastitis. For some pathogens, for example, Salmonella Dublin (Nielsen et al., 2004) and Mycobacterium avium spp. paratuberculosis (Van Schaik et al., 2005), fecal shedding plays an important role in disease dynamics. The identification and removal of carriers is used in control strategies for these pathogens. For other pathogens such as Listeria monocytogenes (Nightingale et al., 2004) or Streptococcus uberis (Zadoks et al., 2005), fecal shedding is not persistent and its role in disease transmission is not clear. The aim of this study was to explore whether fecal shedding of Klebsiella pneumoniae by dairy cows is intermittent, suggesting transient presence or passthrough of organisms, or persistent, which would suggest a gastrointestinal carrier state. To this end, shedding patterns were analyzed with probabilistic methods, associations between shedding status and host factors were evaluated with statistical methods, and heterogeneity of fecal Klebsiella isolates was determined with molecular methods.

A 5-mo longitudinal study was conducted from June through October 2005 on a commercial dairy farm in upstate New York, with approximately 1,200 Holstein milking cows housed in a freestall facility. Two cohorts of 50 cows each were selected from two 200-cow pens with sand bedding. The cows were selected based on the expectation that they would be in the same mid to late lactation group for the next 5 mo. Individual fecal samples from each cow were collected once a month directly from the rectum using individual palpation sleeves (Munoz et al., 2006). Samples were transported in cooler boxes with ice packs and arrived at the laboratory within $1 \mathrm{~h}$ after the 3 -h collection ended. Samples were evaluated for presence of Klebsiella spp. within $24 \mathrm{~h}$ of arrival (Munoz et al., 2006). Briefly, a 1:10 dilution of fecal matter in saline was incubated for $4 \mathrm{~h}$ at $37^{\circ} \mathrm{C}$, and streaked onto MacConkey agar containing $10 \mathrm{mg} / \mathrm{L}$ of ampicillin. Species identity of colonies with Klebsiella morphology was confirmed using citrate, motility, and indole testing (National Mastitis Council, 1999). A cow was considered to shed Klebsiella if at least 1 colony of Klebsiella was detected.

Shedding patterns were evaluated to determine whether shedding of Klebsiella could be described by a 
purely random binomial process, or whether extrabinomial variation was present. The latter would suggest that some cows were more likely to consistently shed Klebsiella than others, potentially due to carrier and noncarrier states. The binomial distribution was given by:

$$
P\left(F S^{+} ; n, p\right)=\frac{n !}{F S^{+} !\left(n-F S^{+}\right) !} p^{F S^{+}}(1-p)^{n-F S^{+}}
$$

where $\mathrm{FS}^{+}$is the number of fecal samples testing positive for Klebsiella (ranging from 0 to 5 ), $n$ is the number of samples for an individual cow, $p$ is the prevalence of shedding, and $P\left(F S^{+}\right)$is the probability of finding $F S^{+}$ positive samples for a cow. Observed and expected values for the number of Klebsiella-positive samples per cow were cross tabulated and the differences between observed and expected values were evaluated using Fisher's exact test. The goals were to determine the probability of finding a specified number of positive samples and the probability of finding specific patterns of shedding. The prevalence of fecal shedding within the cohort differed numerically between samplings (Table 1). As a result, the probability of finding a specified number of positive samples differed between patterns. For example, 3 positive samples could be found in the combinations $(\mathrm{P}, \mathrm{P}, \mathrm{N}, \mathrm{P}, \mathrm{N})$ and $(\mathrm{N}, \mathrm{N}, \mathrm{P}, \mathrm{P}, \mathrm{P})$, where $P$ represents a positive sample and $\mathrm{N}$ represents a negative sample. The expected frequency of the first pattern would be $[0.76 \times 0.77 \times(1-0.86) \times 0.82 \times(1-0.84)]=$ $1 \%$, and the expected frequency of the second pattern would be $[(1-0.76) \times(1-0.77) \times 0.86 \times 0.82 \times 0.84)=3 \%$.

Data on DIM, milk yield, SCC, and parity were collected from herd records maintained in DairyComp 305 (Valley Agricultural Software, Tulare, CA). Data on DIM, yield, and SCC from test days were compared with fecal shedding status for the sampling that was closest in time, either before or after the test day. The distribution of continuous variables (log transformed SCC, DIM, milk yield) was explored using basic descriptive statistics to identify outliers and nonlinearity of associations. Differences in cow factors were compared between Klebsiella-positive and negative animals using 2 -sided 2 -sample $t$-tests. Parity was treated as a categorical variable and associations between shedding status and parity were tested using Pearson's $\chi^{2}$ statistic. Statistical analyses were conducted with Splus version 6.2 (Insightful Corp., Seattle, WA). Significance level was set at $P<0.05$.

Fecal samples were collected from the rectum of a convenience sample of 10 healthy dairy cows in 4 herds (A, B, C, and D). Samples were processed as described above. Up to 4 colonies per fecal sample were selected for confirmation as Klebsiella. The number of samples
Table 1. Observed and expected frequency of Klebsiella shedding patterns in monthly fecal samples from 92 dairy cows ${ }^{1}$

\begin{tabular}{|c|c|c|c|c|c|c|}
\hline \multicolumn{5}{|c|}{ Sampling } & \multicolumn{2}{|c|}{ Pattern frequency ${ }^{2}$} \\
\hline 1 & 2 & 3 & 4 & 5 & Observed & Expected $^{3}$ \\
\hline+ & - & - & - & - & 1 & 0 \\
\hline- & + & + & - & - & 1 & 0 \\
\hline+ & + & + & - & - & 1 & 1 \\
\hline - & + & - & + & - & 1 & 0 \\
\hline+ & + & - & + & - & 0 & 1 \\
\hline- & - & + & + & - & 1 & 1 \\
\hline+ & - & + & + & - & 2 & 2 \\
\hline - & + & + & + & - & 2 & 2 \\
\hline+ & + & + & + & - & 5 & 6 \\
\hline+ & + & - & - & + & 2 & 1 \\
\hline- & - & + & - & + & 2 & 1 \\
\hline+ & - & + & - & + & 1 & 2 \\
\hline - & + & + & - & + & 2 & 2 \\
\hline+ & + & + & - & + & 7 & 7 \\
\hline - & - & - & + & + & 2 & 0 \\
\hline+ & - & - & + & + & 2 & 2 \\
\hline - & + & - & + & + & 1 & 2 \\
\hline+ & + & - & + & + & 4 & 5 \\
\hline - & - & + & + & + & 0 & 3 \\
\hline+ & - & + & + & + & 10 & 10 \\
\hline- & + & + & + & + & 10 & 10 \\
\hline+ & + & + & + & + & 35 & 32 \\
\hline
\end{tabular}

${ }^{1}$ Patterns that were not observed and for which $<1$ cow was expected are not shown; plus and minus signs indicate positive and negative test results, respectively.

${ }^{2}$ Observed and expected values add to 92 and 90 , respectively. Differences in total numbers are due to rounding off of expected values to whole numbers and omission of patterns with $<1$ expected animals and zero observed animals.

${ }^{3}$ Expectation based on assumption of independence of observations and shedding prevalence of $76,77,86,82$, and $85 \%$, respectively, at samplings 1 through 5 .

yielding 4 confirmed Klebsiella colonies for subsequent strain typing ranged from 1 to 6 per herd. In 1 herd (D), longitudinal follow up was performed for 2 cows (D1 and D2) that were Klebsiella positive upon initial screening. Additional samples were collected from cows $\mathrm{D} 1$ and D2 for $5 \mathrm{~d}$ consecutively, 3 wk after the initial sampling, and processed in the same manner as the fecal samples from herds A through D. Klebsiella isolates were further characterized by random amplified polymorphic DNA (RAPD) PCR using primer set AP4/ ERIC-1R: 5'-TCACGATCGA-3'/5'-ATGTAAGCTCCTGGGGATTCGC-3' (Barbier et al., 1996), or ERIC-2/ ERIC-1026: 5'-AAGTAAGTGACTGGGGTGAGCG-3'/ 5'- TACATTCGAGGACCCCTAAGTG-3' (Vogel et al., 1999). Primers were obtained from Integrated DNA Technologies (Coralville, IA). Lysates for PCR were prepared using 10-min boil preparation of cell pellets suspended in $1 \times$ Tris-EDTA buffer (Promega, Madison, WI). Cycling conditions for primer sets AP4/ERIC-1R were initial denaturation for $4 \mathrm{~min}$ at $94^{\circ} \mathrm{C} ; 44$ cycles of $30 \mathrm{~s}$ at $94^{\circ} \mathrm{C}, 1 \mathrm{~min}$ at $35^{\circ} \mathrm{C}$, and $2 \mathrm{~min}$ at $72^{\circ} \mathrm{C}$; and final extension for $7 \mathrm{~min}$ at $72^{\circ} \mathrm{C}$ (Barbier et al., 1996). 
For primer set ERIC-2/1026, 2 initial cycles of $5 \mathrm{~min}$ at $94^{\circ} \mathrm{C}, 5 \mathrm{~min}$ at $35^{\circ} \mathrm{C}$, and $5 \mathrm{~min}$ at $72^{\circ} \mathrm{C}$ were followed by 31 cycles of $1 \mathrm{~min}$ at $94^{\circ} \mathrm{C}, 1 \mathrm{~min}$ at $60^{\circ} \mathrm{C}$, and $2 \mathrm{~min}$ at $72^{\circ} \mathrm{C}$; and a final extension cycle of $8 \mathrm{~min}$ at $72^{\circ} \mathrm{C}$ (Vogel et al., 1999). Electrophoresis was performed in $1.5 \%$ agarose gels (Promega) with 20 wells of $5 \mathrm{~mm}$ width run for $1.5 \mathrm{~h}$ in $0.5 \times$ Tris-borate-EDTA buffer (OmniPur, EMD Chemicals, Gibbstown, NJ) using a horizontal electrophoresis system (D2 Spider, Owl Separation Systems, Portsmouth, NH) at 105 V (EC105 Power Supply, Thermo Electron Corporation, Milford, MA). To allow for assessment of within-sample heterogeneity of Klebsiella strains, isolates originating from 1 sample were always run in adjacent lanes on a gel. Gels were stained with ethidium bromide and images of banding patterns were acquired with the Molecular Imager Gel Doc XR System (BioRad, Hercules, CA) and Quantity One software (version 4.4.1). Each DNA amplification pattern was read by 2 independent examiners who observed the number of DNA fragments and their size relative to a size standard (Trackit 100-bp DNA Ladder, Invitrogen, Carlsbad, CA). Isolates with the same number and size of DNA fragments were considered to belong to the same strain, irrespective of band intensity of fragments. In case of disagreement between observers, RAPD analysis was repeated starting with new lysates to resolve doubts or discrepancies in interpretation.

Ninety-two cows completed 5 samplings. The prevalence of Klebsiella in fecal samples from these cows was $76,77,86,82$, and $85 \%$ for the first, second, third, fourth, and fifth sampling, respectively. The overall prevalence of Klebsiella in fecal samples was $81 \%$. All cows tested positive for Klebsiella at least once, and 35 cows tested positive on all samplings. Thirty-six, 13, 7, and 1 cow(s) tested positive for Klebsiella on 4, 3, 2, and 1 occasion(s), respectively. Observed frequencies did not differ significantly $(P=0.55)$ from those expected based on a random binomial distribution. Animals that were positive more than once could test positive on consecutive samplings or intermittently (Table 1). The observed frequency of shedding patterns was very similar to the frequency that could be expected based on the prevalence of positive animals per sampling and the assumption that results of consecutive samples within cows were independent (Table 1). Observations on 102 cows that were in the cohort for part or all of the study were included in the analysis of host factors. No association was observed between presence or absence of fecal shedding of Klebsiella and DIM, SCC, milk yield, or parity.

The number of Klebsiella strains identified per fecal sample ranged from 2 to 4 and differed among herds
Table 2. Number of Klebsiella strains per fecal sample based on random-amplified polymorphic DNA typing with 2 primer sets (AP4/ ERIC-1R and ERIC-2/1026) ${ }^{1}$

\begin{tabular}{lccc}
\hline & & \multicolumn{2}{c}{ Strains identified } \\
\cline { 3 - 4 } Herd & Sample & AP4/ERIC-1R & ERIC-2/1026 \\
\hline A & 1 & 2 & 2 \\
& 2 & 2 & 2 \\
& 3 & 2 & 2 \\
& 4 & 3 & 3 \\
& 5 & 3 & 3 \\
Average & 6 & 2.3 & 3 \\
B & 1 & 3 & 2.5 \\
& 2 & 3 & 3 \\
Average & & 3 & 3 \\
C & 1 & 4 & 3 \\
& 2 & 4 & 4 \\
Average & 3 & 3.7 & 4 \\
D & & 4 & 4 \\
\hline
\end{tabular}

${ }^{1}$ Four isolates were characterized per sample.

(Table 2). Primer set ERIC-2/1026 discriminated more strains than primer set AP4/ERIC-1R (Table 2, Figure 1). Eight isolates from 3 herds that were indistinguishable after typing with AP4/ERIC-1R were differentiated into 5 patterns with ERIC-2/1026. Primer set AP4/ ERIC-1R did not discriminate any strains that were not also identified by primer set ERIC-2/1026. For 2 cows (D1 and D2), isolates were available from multiple consecutive days and strain heterogeneity was observed within and between samples (Figure 2). For cow D2, up to 4 strains were identified per sample. Each strain was only detected on $1 \mathrm{~d}$. In total, 9 strains were detected among 13 isolates. Cow D1 had up to 3 strains identified per sample. In total, 11 strains were detected amongst 14 isolates, and 9 of 11 strains were only identified on $1 \mathrm{~d}$. The strains identified on initial screening of cow D1 (Table 2) did not match any of the strains identified in subsequent samples.

Klebsiella mastitis management has become an increasing problem, even in herds with inorganic bedding and good environmental hygiene (Munoz et al., 2006). If persistent fecal carriers of Klebsiella could be identified and managed separately, this might provide an additional tool for control of Klebsiella in dairy herds. The fecal shedding patterns observed in our study matched patterns that could be expected based on a fully random binomial distribution. Therefore, the data provided no evidence for a persistent carrier status. Strain-typing data suggest transient presence of Klebsiella rather than persistence, as evidenced by the large variety of Klebsiella strains found within samples, and between samples collected from individual animals on multiple consecutive days. Rapid turnover of the gastrointestinal population has previously been described for $E$. coli 

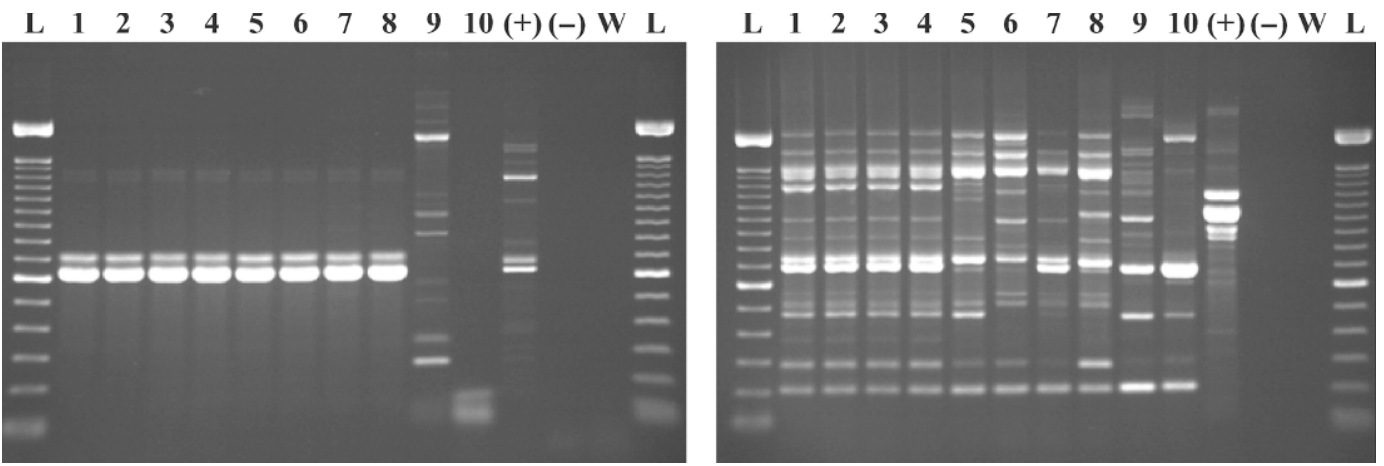

Figure 1. Comparison of random amplified polymorphic DNA typing results for a set of 10 isolates of Klebsiella from bovine feces with primers AP4/ERIC-1R (left) and ERIC-2/1026 (right), respectively. Both gels show the same isolates in the same order. Numbers indicate isolates; $\mathrm{L}=$ DNA ladder; $(+)=$ positive control lysate; $(-)=$ negative control lysate; $\mathrm{W}=$ water.

(Hinton et al., 1985). Such turnover would require frequent ingestion of coliform bacteria. In preliminary analyses in our laboratory, presence of Klebsiella could be shown in feed refusals and water troughs on dairy farms. Fecal excretion of Klebsiella, resulting in contamination of feed and water, and subsequent reingestion of the organism could create an oro-fecal transmission cycle within herds. Additional sources of coliform bacteria may exist in the environment. An association between host factors and fecal shedding of Klebsiella was not detected. This may be due to the relative homogeneity of the population under study, which limited the power of the analysis. Alternatively or additionally, absence of a statistical association may reflect absence of a biological association between fecal shedding of Klebsiella by healthy dairy cows and cow characteristics or production parameters.

Whether fecal strains of Klebsiella have the ability to cause mastitis remains to be established (Munoz et al., 2006). The large heterogeneity of strains found in feces poses a particular challenge in this regard. Considerable resources would be required to characterize the population of fecal Klebsiella isolates in a herd, and to compare the fecal population to isolates from mastitic cows. The RAPD-PCR method used was a simple, affordable, and fast molecular screening tool for withinsample and small-scale within-herd analyses, especially when PCR was performed with primer set ERIC-

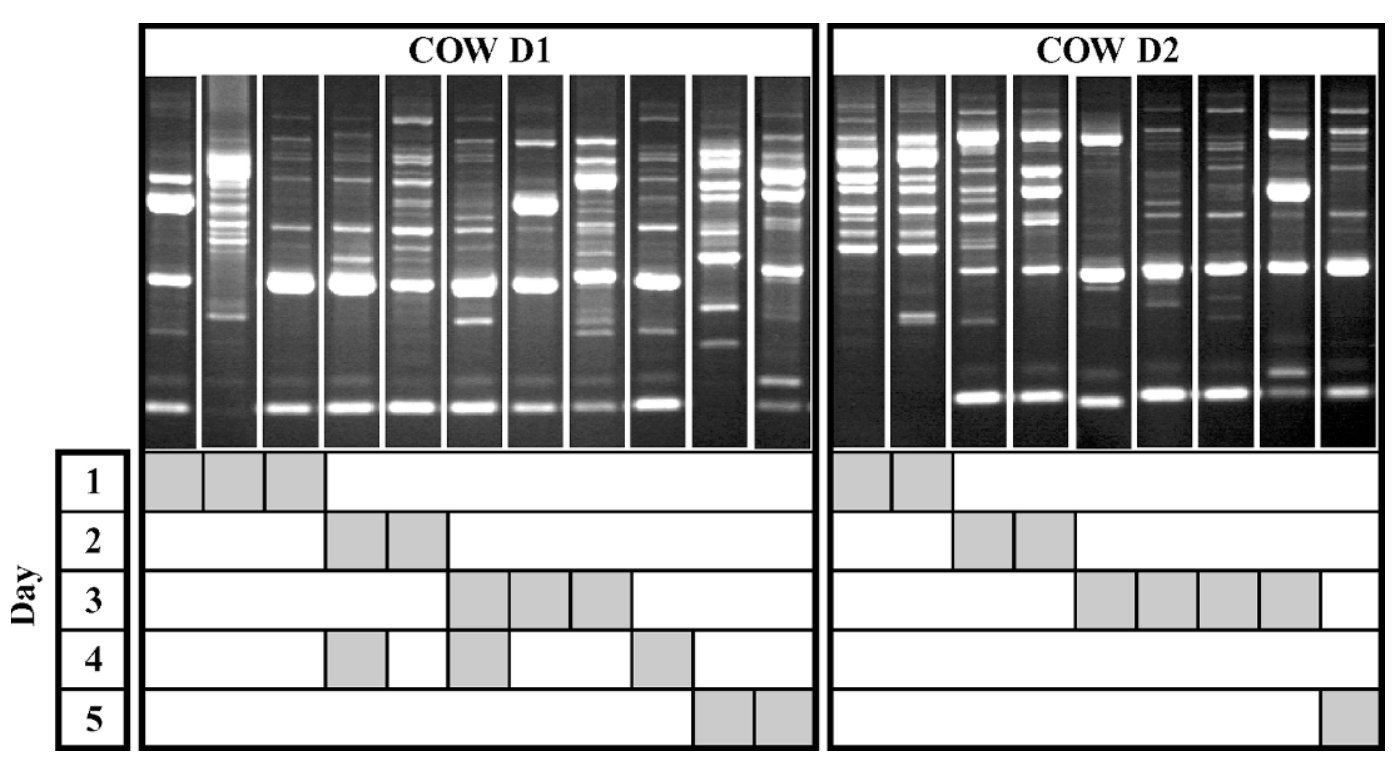

Figure 2. Diversity of Klebsiella strains from fecal samples of 2 cows (D1 and D2) based on banding patterns generated by random amplified polymorphic DNA typing with primer set ERIC-2/1026. Each strain is represented by a banding pattern. Samples were collected on 5 consecutive days (indicated by numbers). Shaded blocks indicate that the specified strain was detected on the specified day. 
2/1026. Complete standardization of RAPD-PCR typing is difficult, and differences in banding patterns do not provide a quantitative measure of genetic differences between strains. This may limit the usefulness of RAPD typing for comparisons of large populations of isolates obtained over extensive time periods or geographic areas (Struelens et al., 1998). For such analyses, a librarytyping method such as multi-locus sequence typing should be used (Struelens et al., 1998; Diancourt et al., 2005). Use of multi-locus sequence typing would increase the time and funding needed for isolate characterize considerably.

In conclusion, Klebsiella appears to be shed randomly and often intermittently rather than persistently by dairy cows, and associations between host factors and shedding status were not detected in a cohort of lactating dairy cattle in the herd under study. Management strategies targeting individual animals can therefore not be proposed based on this study. A large variety of Klebsiella strains can be detected within and between fecal samples, including samples collected from individual animals over multiple consecutive days. The RAPDPCR technique is a useful tool for assessment of strain heterogeneity within samples, but library-typing methods will be needed to compare fecal Klebsiella populations to those associated with mastitis. If fecal and milk isolates constitute overlapping populations, further investigation of methods to reduce fecal shedding are warranted. Because of the intermittent shedding at the animal level, herd- or group-level management strategies, possibly based on nutrition, vaccination, or interruption of an oro-fecal transmission cycle, may be of more interest than control measures at the cow level.

\section{ACKNOWLEDGMENTS}

We thank the participating producers and our colleagues and students at Quality Milk Production Services, in particular Brad Rauch and Christina Ahlstrom, for help with sampling and testing, and Ynte Schukken for advice on preparation of the manuscript.

\section{REFERENCES}

Barbier, N., P. Saulnier, E. Chachaty, S. Dumontier, and A. Andremont. 1996. Random amplified polymorphic DNA typing versus pulsed-field gel electrophoresis for epidemiological typing of vancomycin-resistant enterococci. J. Clin. Microbiol. 34:1096-1099.

Diancourt, L., V. Passet, J. Verhoef, P. A. Grimont, and S. Brisse. 2005. Multilocus sequence typing of Klebsiella pneumoniae nosocomial isolates. J. Clin. Microbiol. 43:4178-4182.

Gröhn, Y. T., D. J. Wilson, R. N. González, J. A. Hertl, H. Schulte, G. Bennett, and Y. H. Schukken. 2004. Effect of pathogen-specific clinical mastitis on milk yield in dairy cows. J. Dairy Sci. 87:3358-3374.

Hinton, M., A. H. Linton, and A. J. Hedges. 1985. The ecology of Escherichia coli in calves reared as dairy-cow replacements. J. Appl. Bacteriol. 58:131-138.

Hogan, J. S., and K. L. Smith. 2003. Coliform mastitis. Vet. Res. 34:507-519.

Kristula, M. A., W. Rogers, J. S. Hogan, and M. Sabo. 2005. Comparison of bacteria populations in clean and recycled sand used for bedding in dairy facilities. J. Dairy Sci. 88:4317-4325.

Munoz, M. A., C. Ahlstrom, B. J. Rauch, and R. N. Zadoks. 2006. Fecal shedding of Klebsiella pneumoniae by dairy cows. J. Dairy Sci. 89:3425-3430.

National Mastitis Council. 1999. Laboratory Handbook on Bovine Mastitis. National Mastitis Council, Madison, WI.

Nielsen, L. R., Y. H. Schukken, Y. T. Gröhn, and A. K. Ersbøll. 2004. Salmonella Dublin infection in dairy cattle: Risk factors for becoming a carrier. Prev. Vet. Med. 65:47-62.

Nightingale, K. K., Y. H. Schukken, C. R. Nightingale, E. D. Fortes, A. J. Ho, Z. Her, Y. T. Grohn, P. L. McDonough, and M. Wiedmann. 2004. Ecology and transmission of Listeria monocytogenes infecting ruminants and in the farm environment. Appl. Environ. Microbiol. 70:4458-4467.

Roberson, J. R., L. D. Warnick, and G. Moore. 2004. Mild to moderate clinical mastitis: Efficacy of intramammary amoxicillin, frequent milk-out, a combined intramammary amoxicillin, and frequent milk-out treatment versus no treatment. J. Dairy Sci. 87:583-592.

Struelens, M. J., Y. De Gheldre, and A. Deplano. 1998. Comparative and library epidemiological typing systems: Outbreak investigations versus surveillance systems. Infect. Control Hosp. Epidemiol. 19:565-569.

Van Schaik, G., S. M. Stehman, R. H. Jacobson, Y. H. Schukken, S. J. Shin, and D. H. Lein. 2005. Cow-level evaluation of a kinetics ELISA with multiple cutoff values to detect fecal shedding of Mycobacterium avium subspecies paratuberculosis in New York State dairy cows. Prev. Vet. Med. 72:221-236.

Vogel, L., G. Jones, S. Triep, A. Koek, and L. Dijkshoorn. 1999. RAPD typing of Klebsiella pneumoniae, Klebsiella oxytoca, Serratia marcescens and Pseudomonas aeruginosa isolates using standardized reagents. Clin. Microbiol. Infect. 5:270-276.

Zadoks, R. N., L. L. Tikofsky, and K. J. Boor. 2005. Ribotyping of Streptococcus uberis from a dairy's environment, bovine feces, and milk. Vet. Microbiol. 109:257-265. 